\title{
openheart Presentation and outcomes of mitral valve surgery in France in the recent era: a nationwide perspective
}

\author{
David Messika-Zeitoun (D) , ${ }^{1}$ Pascal Candolfi, ${ }^{2}$ Maurice Enriquez-Sarano, ${ }^{3}$ \\ Ian G Burwash, ${ }^{1}$ Vincent Chan, ${ }^{4}$ Jean-Francois Philippon, ${ }^{5}$ \\ Jean-Manuel Toussaint, ${ }^{6}$ Partrick Verta, ${ }^{7}$ Ted E Feldman, ${ }^{7}$ Bernard lung, ${ }^{8}$ \\ David Glineur, ${ }^{4}$ Jean-Francois Obadia, ${ }^{9}$ Alec Vahanian, ${ }^{10}$ Thierry Mesana ${ }^{4}$
}

To cite: Messika-Zeitoun $D$, Candolfi P, Enriquez-Sarano M, et al. Presentation and outcomes of mitral valve surgery in France in the recent era: a nationwide perspective. Open Heart 2020;7:e001339. doi:10.1136/ openhrt-2020-001339

Received 12 May 2020 Revised 8 July 2020 Accepted 8 July 2020

Check for updates

(C) Author(s) (or their employer(s)) 2020. Re-use permitted under CC BY-NC. No commercial re-use. See rights and permissions. Published by BMJ.

For numbered affiliations see end of article.

Correspondence to Dr David Messika-Zeitoun; dmessika-zeitoun@ottawaheart. ca

\section{ABSTRACT}

Objectives Unbiased information regarding the surgical management of patients with mitral regurgitation (MR) at the nationwide level are scarce and mainly US-based. The Programme de Médicalisation des Systèmes d'Information, a mandatory national database, offers the unique opportunity to assess the presentation and outcomes of all consecutive mitral valve (MV) surgeries performed in France in the contemporary era.

Methods We collected all MV surgeries performed for MR in France in 2014-2016. MR aetiology was classified as degenerative (DMR), secondary (SMR) or Other (rheumatic or congenital disease and infective endocarditis).

Results During the 3-year period, $18167 \mathrm{MV}$ surgeries were performed in France (55\% repair and 45\% replacement; $52 \%$ isolated). Age was $66 \pm 12$ years and $59 \%$ were male. Aetiology was DMR in $42 \%$, SMR in $16 \%$ and other in $42 \%$ including $19 \%$ with uncertain aetiologies. Overall, in-hospital mortality was $6.5 \%$ and increased with age, female gender, Charlson Comorbidity Index, type of surgery (replacement vs repair), associated surgery (combined vs isolated) and MR aetiology (all $p<0.01$ ). Inhospital mortality and rate of death/readmission for heart failure (HF) at 1 year were $3.4 \%$ and $13 \%$, respectively for DMR (2.4\% and $11 \%$ for isolated DMR) and $7.8 \%$ and $27 \%$, respectively for SMR $(5.5 \%$ and $23 \%$ for isolated SMR). Repair rate was $55 \%$ overall, $68 \%$ in DMR and $72 \%$ for isolated DMR surgery (70\% of all DMR). Repair rates decreased with age, Charlson Comorbidity Index and female sex (all $p<0.0001$ ).

Conclusion In this cross-sectional contemporary prospective nationwide database, in-hospital mortality and 1 year rate of death and HF readmission were considerable overall and in all subsets. Repair rates were suboptimal overall especially in the elderly and women subsets. These results underline the need to develop strategies to improve management and outcomes of patients with both DMR and SMR.

\section{INTRODUCTION}

Mitral regurgitation (MR) is the most common valve disease in Western countries and its prevalence is expected to dramatically increase with the ageing of the population. ${ }^{1}$

\section{Key questions}

What is already known about this subject?

- Leading mitral valve centres have reported generally very high mitral repair rates with extremely low operative mortality but unbiased information regarding the surgical management and outcomes of patients with mitral regurgitation (MR) at the nationwide level are rare and mainly US-based.

What does this study add?

- Using the Programme de Médicalisation des Systèmes d'Information, an exhaustive national database, we recorded all consecutive cases of mitral valve surgeries performed in France in 2014-2016 and examined their presentation and outcomes.

- Operative mortality and 1-year rate of death and readmission for heart failure were considerable overal and in all subsets suggesting that patients are still referred at an advanced disease stage.

- In addition, repair rates were suboptimal overall and in patients with isolated degenerative MR, especially in the elderly and women subsets as well as in patients who underwent a combined procedure.

How might this impact on clinical practice?

- There is a critical need to develop strategies to improve management and outcomes of patients with both degenerative and secondary MR.

MR is usually classified as primary led by degenerative aetiologies (DMR) or as secondary (SMR) in which the valve components are structurally normal. ${ }^{2} \mathrm{MR}$ is a serious condition associated with excess mortality which is unfortunately often undertreated especially in older patients. ${ }^{3} 4$ Even when offered surgery, patients' outcomes remain mediocre if the intervention is not timely performed.

Series reporting the results of surgical mitral valve (MV) interventions are numerous but are usually retrospective and/ or single-centre. In addition to in-hospital 
mortality, these series also report repair rates in DMR that is uniformly superior to replacement among all age ranges. ${ }^{6-10}$ The benefit of MV repair over replacement in SMR remains debated and exposes to a significant risk of MR recurrence. ${ }^{11-14}$ The performance of MV repair is often considered as the hallmark of quality of surgical treatment of DMR and a quality indicator to assess and benchmark surgical institutions. ${ }^{15}$ Leading MV centres have reported generally very high mitral repair rates with extremely low operative mortality, ${ }^{16-18}$ but reports from these highly selected centres suffer from obvious selection bias in terms of both patient referral and operators/ teams skills questioning their generalisability to the real world.

Contemporary, exhaustive and unbiased information regarding the surgical management and outcomes of patients with MR at the nationwide level are critical for policy makers to develop efficient programmes to improve management of these patients. Most of the literature is US-based (eg, the Society of Thoracic Surgeons (STS) or the New York State databases), which rely on a very different healthcare system than most European

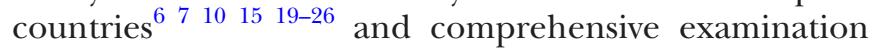
of mitral surgery in routine practice in European countries are scarce. ${ }^{27}$ The Programme de Médicalisation des Systèmes d'Information (PMSI) is an exhaustive national database that records all consecutive cases of MV surgeries performed in France, as it is mandatory to all French healthcare institution. The PMSI offers the unique opportunity to evaluate real-life outcomes after MV surgery at the nationwide level.

\section{METHODS \\ Study design}

All healthcare institutions in France are mandated to transfer information regarding their activity into the PMSI database. The PMSI dataset includes information about the patient (age and sex), the hospital, the admission (date of admission and of discharge), pathologies, procedures and in-hospital outcome. ${ }^{28}$ Primary and secondary diagnoses are coded using the 10th revision of the International Statistical Classification of Diseases and Related Health Problems (ICD-10). Procedures are coded using a French standardised classification. ${ }^{29}$

We collected all cases of MV surgery (isolated or combined with other procedures) performed on native MV in 2014-2016 in adults (age $\geq 30$ years) both in public and private hospitals in France. Our study population was identified using procedure codes for MV surgery (DBKA 002/005/010 and DBMA 002/003/005/007/013) along with the ICD-10 codes for mitral regurgitation (I340 and I341). Patients with mitral stenosis or mixed disease were excluded. According to ICD-10 codes and associated diseases/interventions, the population was divided into three groups, DMR, SMR and other (rheumatic valve disease or infective endocarditis, congenital diseases and combined pulmonary valve intervention). In the
DMR group, patients had to be free from prior history of ischaemic/dilated cardiomyopathy, coronary disease, myocardial infarction and coronary artery bypass graft or other cardiac (CABG) surgery. Patients who underwent a CABG at the time of the MV surgery who likely represented patients diagnosed with coronary artery disease on the preoperative angiogram were not excluded from the DMR group. In contrast, patients with SMR had to have at least one of the above-mentioned associated conditions in their medical history. Patients were subclassified based on whether a combined procedure was performed at the same time (combined or isolated MV surgery). Type of surgery (replacement vs repair) was also recorded. The Charlson Comorbidity Index was used to assess for patients' comorbidities. ${ }^{30}$

\section{Outcome}

In-hospital mortality was defined as death occurring between the intervention and hospital discharge during the same hospital stay. Total length of stay (LOS) was calculated as the time duration between the admission and hospital discharge and expressed in days. LOS in the intensive care unit (ICU) was calculated as the time duration between the admission to the ICU and discharge from the ICU and expressed in days. PMSI was used to collect in-hospital death as well as 1 year rates of death and readmission for heart failure. As the PMSI only captures admission, out-of-hospital events were not included in the present manuscript.

\section{Statistics}

Continuous variables were expressed as mean $\pm \mathrm{SD}$ or median $(95 \% \mathrm{CI})$ and categorical variable as number of patients (percentage). Differences between groups were calculated with the use of the $\chi^{2}$ test for categorical variables and Student's t-test or Wilcoxon/Kruskall-Wallis tests for continuous variables as appropriate. Variables associated with in-hospital mortality or repair rates in univariate analysis $(p<0.10)$ were entered into a stepwise multiple linear regression model. A Cox proportional hazard analysis was used to evaluate the predictors of death and readmission for heart failure. All tests were two-sided and performed using JMP V.9.0 (SAS Institute, Cary, North Carolina, USA). A p value $<0.05$ was considered to be statistically significant.

\section{RESULTS \\ Population}

Between January 2014 and December 2016, 18167 MV surgeries were performed in France in adult patients 30 years of age or older in 56 centres (mean yearly MV surgery case volume $108 \pm 56$ (median 104, IQR $(66-143)))$. The mean age of the population was $66 \pm 12$ years and $59 \%$ were male. Mean Charlson Comorbidity Index was $1.49 \pm 2.10$ and 6325 patients $(35 \%)$ had a Charlson Comorbidity Index $\geq 2$. Overall, 7593 patients (42\%) were classified as having DMR and 2935 patients (16\%) having SMR. The other group comprised 4086 


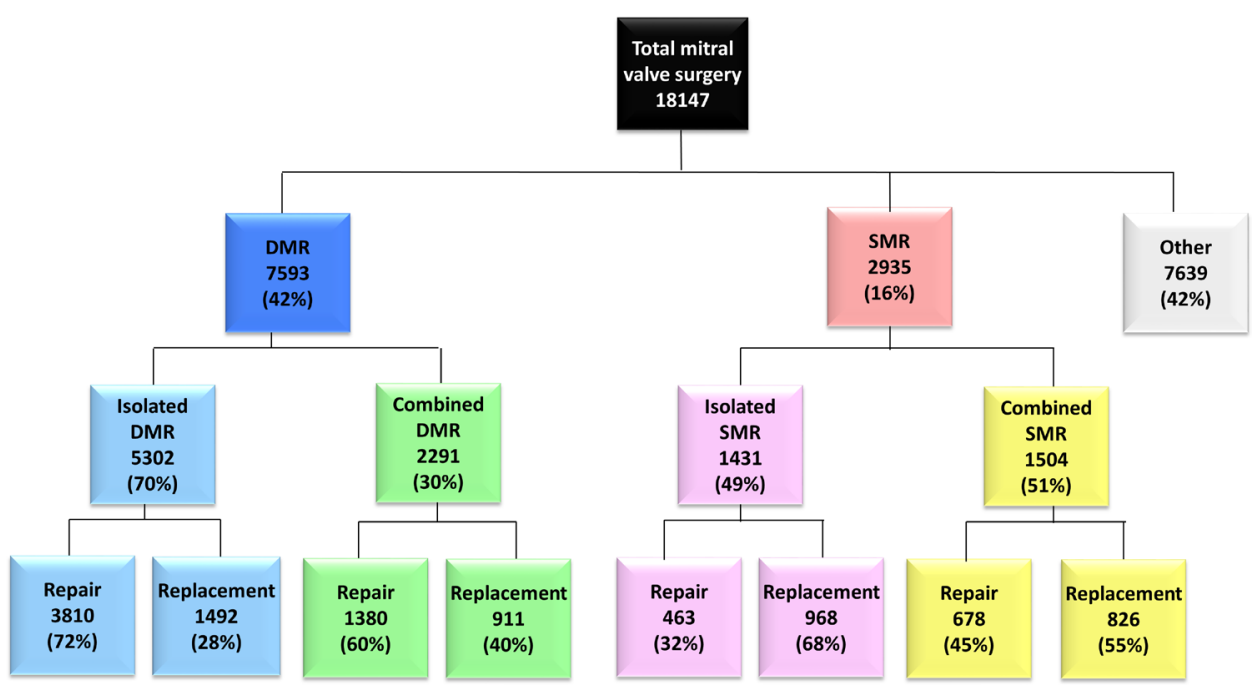

Figure 1 Flow chart of the population. DMR, degenerative mitral regurgitation; SMR, secondary mitral regurgitation.

patients (23\%) with rheumatic/congenital diseases or endocarditis and 3553 patients $(19 \%)$ with uncertain MR aetiology (figure 1). Characteristics and outcomes of the overall population as well as comparison between DMR and SMR are presented in table 1 .

\section{In-hospital mortality and 1-year outcomes Overall}

A MV replacement was performed in 8126 patients $(45 \%)$ and an MV repair in 10041 patients (55\%). Isolated mitral surgery accounted for $52 \%$ of all surgeries (9522 patients). In-hospital mortality was 6.5\% (1183 patients) (figure 2). Mean total and ICU LOS duration were $17 \pm 15$ days (median 13 days, IQR 9-19) and $9 \pm 12$ days (median 6 days, IQR 3-10), respectively. In-hospital mortality increased with age $(3.4 \%$ in patients under 60 years of age, $6.0 \%$ in patients between 60 and 74 years and $10.3 \%$ after 75 years, $\mathrm{p}<0.0001$ ), female gender ( $7.2 \%$ vs $6.1 \%$ in men, $\mathrm{p}=0.0003)$ and Charlson Comorbidity Index $(2.5 \%$ in patients with Charlson Comorbidity Index of $0,5.6 \%$ in patients with Charlson Comorbidity
Index of $1 \%$ and $11.8 \%$ in patients with Charlson Comorbidity Index $\geq 2, p<0.0001)$. In-hospital mortality was also higher in patients who underwent an MV replacement versus repair $(10.8 \%$ vs $3.1 \%, \mathrm{p}<0.0001)$ and a combined versus isolated surgery $(8.9 \%$ vs $4.6 \%$, $\mathrm{p}<0.0001)$. In-hospital mortality was markedly different across MR aetiology, 3.4\% in DMR, $7.8 \%$ in SMR and $9.1 \%$ in other $(\mathrm{p}<0.0001)$. All these parameters but sex $(\mathrm{p}=0.44)$ were independently associated with in-hospital mortality (all $\mathrm{p}<0.0001)$.

Isolated mitral valve surgery

Mean age was $65 \pm 12$ years and 5546 patients (58\%) were male. Among the 9522 patients who underwent an isolated surgery, a repair was performed in 5356 (56\%) and a replacement in 4166 (44\%). In-hospital mortality was $4.6 \%$ and total and ICU LOS were $16 \pm 14$ days (median 12 days, IQR 9-17) and $8 \pm 10$ days (median 5 days, IQR IQR 3-9), respectively. As in the overall population, mortality rates increased with age $(p<0.0001)$, female gender $(\mathrm{p}=0.0009)$, Charlson Comorbidity Index

\begin{tabular}{|c|c|c|c|c|c|}
\hline & Overall & DMR (n=7726) & SMR $(n=3015)$ & $\begin{array}{l}\text { P value between } \\
\text { DMR and SMR }\end{array}$ & $\begin{array}{l}\text { Other/Unclassified } \\
(\mathrm{n}=7639)\end{array}$ \\
\hline Age & $66 \pm 12$ & $66 \pm 12$ & $67 \pm 11$ & $<0.0001$ & $66 \pm 12$ \\
\hline Male gender & $10451(59)$ & $4535(60)$ & $1662(57)$ & 0.004 & $4554(60)$ \\
\hline Charlson Comorbidity Index & $1.49 \pm 2.10$ & $0.83 \pm 1.34$ & $2.01 \pm 2.16$ & $<0.0001$ & $1.95 \pm 2.42$ \\
\hline Charlson Comorbidity Index $\geq 2$ & $6325(35)$ & $1454(19)$ & $1443(43)$ & $<0.0001$ & $3428(45)$ \\
\hline Isolated mitral valve surgery & 9522 (52) & $5302(70)$ & $1431(49)$ & $<0.0001$ & $3429(45)$ \\
\hline Mitral valve repair & $10041(55)$ & $5190(68)$ & $1141(39)$ & $<0.0001$ & $3929(49)$ \\
\hline Public hospital & $12840(71)$ & $4748(67)$ & $2343(80)$ & $<0.0001$ & $5749(75)$ \\
\hline Length of stay, total & $17 \pm 15$ & $14 \pm 9$ & $19 \pm 16$ & $<0.0001$ & $20 \pm 18$ \\
\hline Length of stay, intensive care unit & $9 \pm 12$ & $7 \pm 7$ & $10 \pm 13$ & $<0.0001$ & $10 \pm 14$ \\
\hline In-hospital mortality & $1183(6.5)$ & $258(3.4)$ & $229(7.8)$ & $<0.0001$ & $696(9.1)$ \\
\hline
\end{tabular}

DMR, degenerative mitral regurgitation; SMR, secondary mitral regurgitation. 


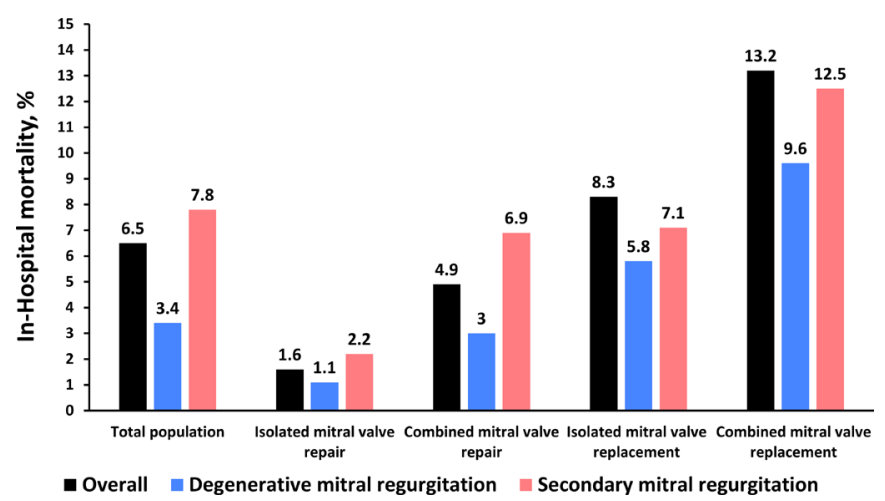

Figure 2 In-hospital mortality according to the type of surgery (replacement versus repair) and aetiology of the mitral regurgitation.

$(\mathrm{p}<0.0001)$, type of surgery (replacement vs repair, $\mathrm{p}<0.0001)$ and MR aetiology $(\mathrm{p}<0.0001)$ and in multivariate analysis all but sex were independently associated with in-hospital mortality (all $\mathrm{p}<0.0001$ ).
Degenerative mitral regurgitation

Surgery was isolated in 5302 patients $(70 \%)$ and combined in 2291 patients $(30 \%)$ with either a tricuspid valve (1496 patients; 20\%), an aortic valve (479 patients; $6 \%$ ) or a CABG surgery (447 patients; 6\%). An MV repair was performed in 5190 patients $(68 \%)$ and an MV replacement in 2403 patients (32\%). Overall, in-hospital mortality was $3.4 \%$ (258 patients). As expected, patients who underwent combined surgery had a more advanced presentation than those who underwent an isolated MV surgery (table 2 and figure 2) and a twice as high mortality rate $(5.6 \%$ vs $2.4 \%, \mathrm{p}<0.0001)$. In both subsets (isolated and combined MV surgery), mortality rates were also markedly higher in patients who underwent an MV replacement versus repair (both $\mathrm{p}<0.0001$, table 2). Importantly, in-hospital mortality of isolated MV repair (49\% of all DMR surgeries) was only $1.1 \%$ (vs $5.8 \%$ in isolated MV replacement, $\mathrm{p}<0.0001)$. Age, Charlson Comorbidity Index, combined surgery and replacement were independently associated with higher mortality (all $\mathrm{p}<0.0001)$, whereas sex was not $(\mathrm{p}=0.95)$. Combined rate

Table 2 Characteristics and outcome according to the type of surgery (mitral valve repair or replacement) and associated surgery (isolated or combined) (A) in the subset of patients with degenerative mitral regurgitation and (B) in the subset of patients with functional mitral regurgitation

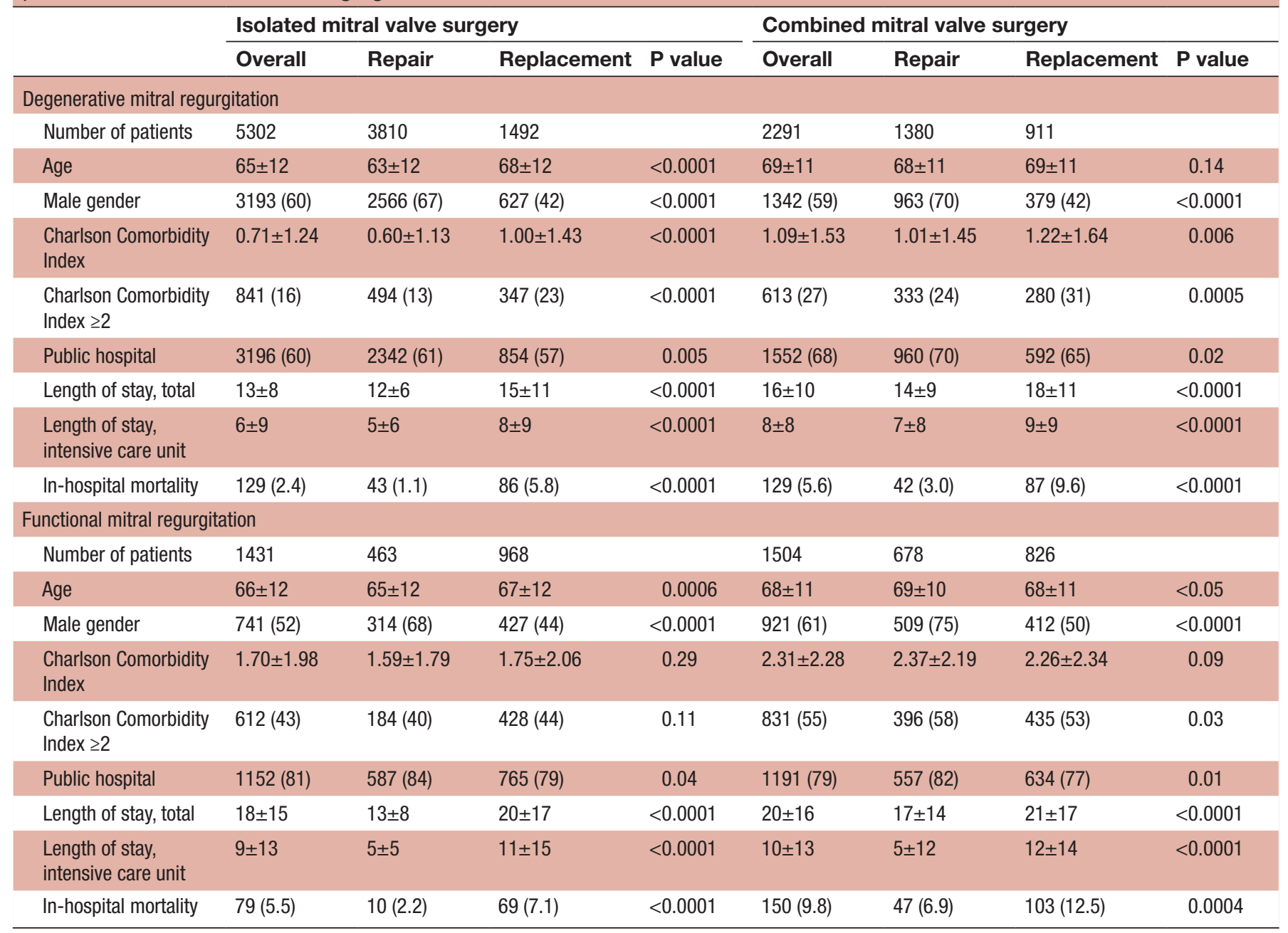



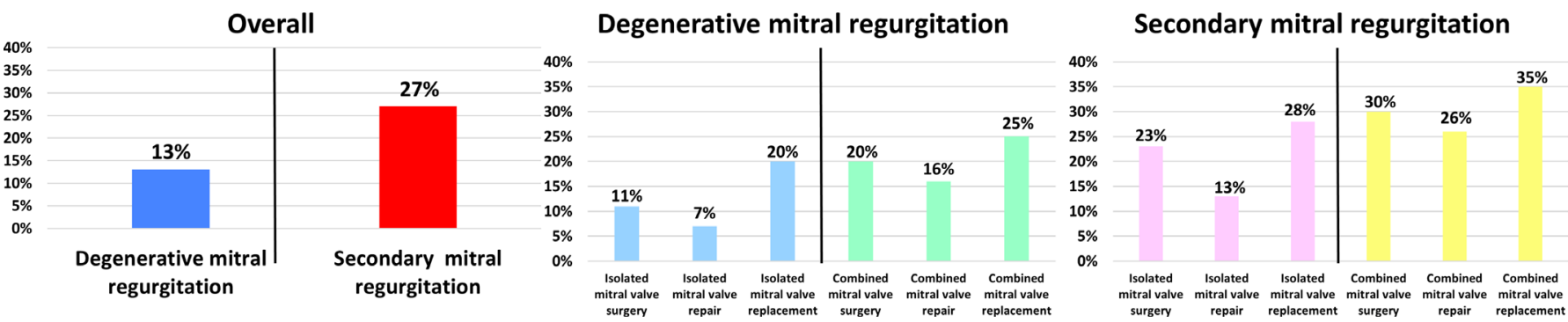

Figure 3 One-year rate of death or readmission for heart failure. (A) Overall in degenerative mitral regurgitation (DMR) and secondary mitral regurgitation (SMR), (B) in DMR and (C) in SMR according to type of surgery (repair or replacement) and performance or not of combined surgery (isolated mitral valve repair, isolated mitral valve replacement, combined mitral valve repair and combined mitral valve replacement).

of death (including in-hospital mortality) and readmission for $\mathrm{HF}$ at 1 year was $13 \%$ overall and increased with the type of surgery (replacement vs repair) and performance of combined surgery (all $\mathrm{p}<0.0001$, figure 3 ).

\section{Secondary mitral regurgitation}

Mean age was $67 \pm 11$ years and 1662 patients $(57 \%)$ were male. An MV repair was performed in 1141 patients $(39 \%)$ and a combined surgery in 1504 patients $(51 \%)$ (tricuspid valve surgery in 792 patients (27\%), CABG in 558 patients $(19 \%)$ and aortic valve surgery in 352 patients (12\%)). Overall, in-hospital mortality was $7.8 \%$ (229 patients) (figure 2) and rate of death and readmission for $\mathrm{HF}$ at 1 year was 27\% (figure 3). As for DMR, mortality rates and rates of death and readmission for $\mathrm{HF}$ at 1 year increased with type of surgery and performance of combined surgery (all $\mathrm{p}<0.0001$, table 2 and figure 3 ).

\section{Repair rates}

\section{Isolated mitral valve surgery}

Repair rate was 56\% overall and decreased with age, sex and Charlson Comorbidity Index. Thus, repair rates were $65 \%$ in patients under 60 years of age, $56 \%$ in patients between 60 and 74 years and $46 \%$ after 75 years $(p<0.0001)$. Repair rate was $65 \%$ in men and $43 \%$ in women $(\mathrm{p}<0.0001)$ and $66 \%$ in patients with Charlson Comorbidity Index of $0,56 \%$ in patients with Charlson Comorbidity Index of $1 \%$ and $39 \%$ in patients with Charlson Comorbidity Index $\geq 2(p<0.0001)$. In multivariate analysis, age, female gender and Charlson Comorbidity Index were inversely associated with performance of an MV repair (all $\mathrm{p}<0.0001$ ).

\section{Degenerative mitral regurgitation}

Repair rate was $68 \%$ overall and was significantly lower in patients who underwent combined versus isolated MV surgery $(60 \%$ vs $72 \%, \mathrm{p}<0.0001)$. Overall, patients who underwent a repair compared with those who underwent a replacement were younger, more frequently men and had lesser burden of comorbidities (table 2). In multivariate analysis, age, female gender, Charlson Comorbidity Index and combined surgery were all independently associated with lower repair rate (all $\mathrm{p}<0.0001)$. Among the 5302 patients who underwent an isolated MV surgery for DMR, repair rates were $72 \%$ overall, $81 \%$ in patients under 60 years of age, $72 \%$ in patients between 60 and 74 years and $59 \%$ after 75 years $(\mathrm{p}<0.0001)$. Repair rates also decreased with increased burden of comorbidities: $77 \%$ in patients with Charlson Comorbidity Index of 0 , $68 \%$ in patients with Charlson Comorbidity Index of 1 and $59 \%$ in patients with Charlson Comorbidity Index $\geq 2(p<0.0001)$. Interestingly, repair rate was also lower in women than in men $(59 \%$ vs $80 \%, \mathrm{p}<0.0001)$. Compared with men, women were older ( $66 \pm 12$ years vs $62 \pm 12$ years, $\mathrm{p}<0.0001)$ and presented with higher Charlson Comorbidity Index $(0.77 \pm 1.26$ vs $0.67 \pm 1.22, \mathrm{p}<0.0001)$ but after adjustment for age and Charlson Comorbidity Index, repair rate remained significantly and independently lower in women than in men $(\mathrm{p}<0.0001)$. The lower repair rate in women in all age and Charlson Comorbidity Index categories is illustrated in figure 4 .

\section{DISCUSSION}

In the present study, we report nationwide data on MV surgery performed in France in the contemporary era. Main results can be summarised as follow. First, in the contemporary era, MV surgery remained associated with a high in-hospital mortality that increased with age, Charlson Comorbidity Index, replacement versus repair, combined versus isolated surgery and MR aetiology. Second, DMR represented the main aetiology leading to MV surgery and was twice as frequent as SMR. DMR surgery was performed more frequently isolated than combined but surprisingly isolated surgery was also performed in approximately half of the patients with SMR. Third, operative mortality and 1-year rate of death and HF readmission were approximately twice as high in SMR as in DMR but overall substantial in both subsets. Fourth, the repair rate remained suboptimal overall and in all subsets including DMR and was only slightly higher when MV surgery was isolated than combined. Elderly and women were less likely to undergo an MV repair. In contrast, most patients with SMR underwent a MV replacement.

\section{Overall results of MV surgery in France}

Our study clearly shows that, in the contemporary era, MV surgery remained associated with a relatively high in-hospital mortality. Main determinants of mortality were age, 
Age $<60$ years

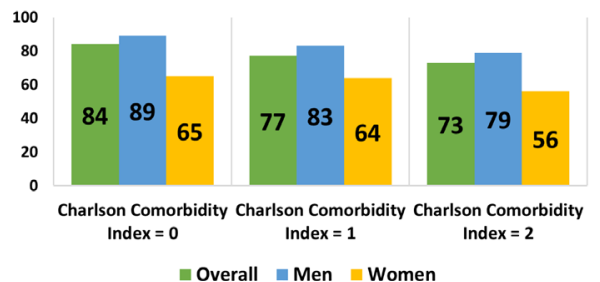

Age 60 - 74 years

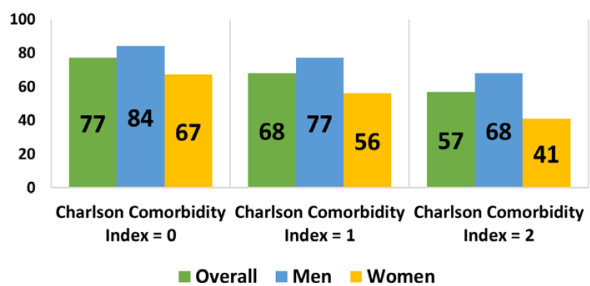

Age $\geq 75$ years

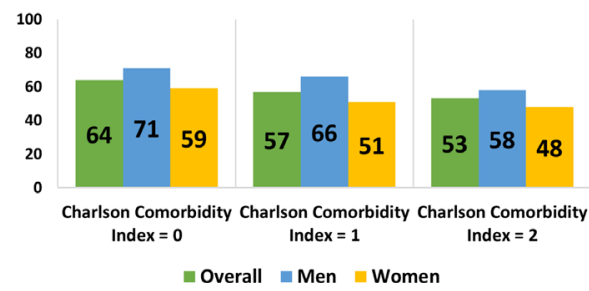

Figure 4 Repair rate overall, in male and in female patients undergoing isolated mitral valve surgery for degenerative mitral regurgitation according to age and Charlson Comorbidity Index.

Charlson Comorbidity Index, type of surgery, combined surgery and MR aetiology. In-hospital mortality rates for isolated MV replacement for both DMR and SMR were $5.8 \%$ and $7.1 \%$, respectively and as high as $12.5 \%$ for combined MV surgery in SMR. One-year mortality and readmission rates for HF remained substantial even in patients who underwent an isolated MV surgery (11\% for DMR and 23\% for SMR).

DMR was the main reason for MV surgery and was twice as common as SMR. This ratio is in agreement with a recent prospective multicentre echocardiographic registry. ${ }^{31}$ Therapeutic approaches for SMR remain a matter of debate and the benefit of surgical correction of SMR is disputed. ${ }^{13} 3233$ Interestingly and despite these uncertainties, half of the patients with SMR underwent an isolated MV surgery, a markedly higher rate than one might have expected. In addition, two-third were mitral replacement, which may represent a trend to avoid the high MR recurrence rates as observed with MV repair in the Cardiothoracic Surgical Trials Network trials. ${ }^{13} 3233$ With the development of transcatheter therapy and the extension of the indications for SMR, this rate is expected to decrease in the following years. ${ }^{34}{ }^{35}$

\section{Repair rates}

The advantages of MV repair over replacement is well established. MV repair offers a lower operating mortality, a higher left ventricular ejection fraction preservation, a lower risk of stroke and infection, the avoidance of anticoagulation and overall a better long-term survival. ${ }^{6-10}$ 36-38 In patients with DMR who underwent an isolated MV surgery, repair rate was overall $72 \%$ and decreased with age and Charlson Comorbidity Index. It is worth noting that these rates compared well with those reported in the USA. $^{6-8} 19$ Repair rate also markedly decreased (60\%) when a combined procedure was performed, although tricuspid valve surgery was the most common intervention. As patients who underwent a combined surgery were older, presented with higher Charlson Comorbidity Index and likely more advanced disease, whether this lower repair rate is related to surgeons' preference or to anatomical differences could not be established. However, given the well-established benefit of repair versus replacement across all subsets, ${ }^{6-10}$ repair should be the choice of technique when the anatomy is suitable.

Prior studies have shown that women were underdiagnosed, under-treated and referred later than men for
MV surgery, which translated into an excess mortality risk compared with men. We could not calculate any surgical risk scores and we used the Charlson Comorbidity Index as a surrogate. In the present study, using a population and nationwide contemporary analysis, we showed that women were less likely to undergo an MV repair than men, although MV replacement carries a higher risk and participate to the poorer outcomes observed in women. Two potential explanations can be proposed: 1) anatomical differences between men and women as a lower rate of posterior leaflet prolapse and more frequent leaflets' calcifications $^{39} 40$ leading to more complex repair and a lower likelihood of MV repair and 2) a sex-based bias in the treatment of DMR cannot be excluded. In this regard, it is worth noting that the repair rate was lower in women than in men in all age and Charlson Comorbidity Index categories including in the youngest subset with no significant comorbidities arguing against the role of anatomic factors in treatment's decision. Furthermore, the lack of impact of sex on mortality after adjustment for the type of mitral surgery does not support a later referral in women than in men. Regardless of the reasons, the lower rate of repair in women is a major concern deserving specific correcting actions.

\section{Clinical implication}

The high mortality and morbidity observed in the present study may suggest that, in the most contemporary era, patients are still often referred late in the course of the disease despite clear opposite recommendations. ${ }^{41}{ }^{42}$ Timely intervention is of utmost importance to reduce the number of patients referred at an advanced disease stage such as with severe symptoms, marked left ventricular remodelling or elevated systolic pulmonary pressure, which directly negatively affect immediate and long-term outcomes. A recent report issued from the STS database showed that among patients referred for an isolated MV surgery in the 2011-2016 period, half of the patients presented a recent history of heart failure, $47 \%$ with a reduced ejection fraction and $24 \%$ with high systolic pulmonary artery pressures. ${ }^{6}$ We could however not assess such parameters from the PMSI database and thus a late referral as potential explanation for the high observed mortality is only speculative. Centres' and surgeons' volume and expertise could also be proposed to explain suboptimal outcomes. ${ }^{43}$ Development of dedicated programmes to raise awareness of valvular heart 
disease, screening programmes and education of care providers regarding clinical guidelines ${ }^{41}{ }^{42}$ are critical to improve timeliness of interventions. ${ }^{44}$ The overall suboptimal repair rates even in the most ideal subset of patients, that is, patients 60 years of age or younger with no comorbidities who underwent an isolated MV surgery for DMR $(84 \%)$ is also of major concern, especially in regard to the $1.1 \%$ mortality that could be achieved in patients who underwent an isolated MV repair. Altogether our results are a strong incentive to the implementation of dedicated referral pathways and the development of Heart Valve Centres of Excellence capable of achieving high repair rate and excellent outcomes ${ }^{45}$ as well as to offer the full armamentarium of mitral interventions including transcatheter therapies.

\section{Strengths and limitations}

The present study deserves several comments. First, there are inherent limitations to the PMSI database such as a limited granularity. Thus, we were limited in our analysis to variables that were present in the database and information regarding the New York Heart Association functional class, ejection fraction or type of surgical prosthesis were not available. We could not calculate any surgical risk scores and we used the Charlson Comorbidity Index as a surrogate. Mortality and readmissions, linked to reimbursement, are expected to be of good quality. Importantly, in contrast to the STS database, which relies on the 'voluntary' participation of both centres and surgeons and also includes Canadian centres, the PMSI is mandatory and we were able to capture all consecutive MV surgeries performed at the nationwide level during the study period. Second, classification of MR aetiology between degenerative and secondary did not rely on anatomic assessment performed at the centre level. However, a very similar methodology was previously used in the New York State database to identify 'presumed' DMR. ${ }^{15}$ It is also worth noting that MR aetiology was also missing in up to one-third of patients in the STS database. ${ }^{6}{ }^{22}$ However, suboptimal repair rates were consistently observed across all subsets including patients who underwent an isolated MV surgery or those classified as DMR. In addition, although we acknowledged as a limitation the absence of clear MR aetiology in 19\% of our population, we weighted specificity over sensitivity for the definition of DMR and inclusion of these patients in the DMR subset would have led to an even lower repair rates and higher in-hospital mortality rates. We also excluded 285 adult patients between 18 and 30 years and possibly congenital MR. Third, only in-hospital death and HF readmission are captured in the PMSI. As out-of-hospital events were not captured, these rates are likely underestimated. Fourth, only the intervention finally performed was collected and repair attempt rate was not available. In addition, the rate of residual MR and repair durability, which are critical, are not captured in the PMSI. Finally, although several coauthors are companies' employees, the first author had full access and independence at all stage of the project from study design, data extraction and analysis to manuscript drafting.

\section{CONCLUSION}

Based on a prospective national database, we report the contemporary presentation and outcomes of all MV surgeries performed in France. Operative mortality and 1-year rate of death and readmission for heart failure were substantial even for isolated DMR, the main aetiology leading to MV surgery. Repair rates were overall suboptimal, especially in the elderly and women subsets as well as in patients who underwent a combined procedure. These results underline the need to develop strategies to improve management and outcomes of patients with both DMR and SMR.

\section{Author affiliations}

${ }^{1}$ Cardiology, University of Ottawa Heart Institute, Ottawa, Ontario, Canada ${ }^{2}$ Edwards Lifesciences, Nyon, Switzerland

${ }^{3}$ Cardiovascular Diseases and Internal Medicine, Mayo Clinic, Rochester, Minnesota, USA

${ }^{4}$ Surgery, University of Ottawa Heart Institute, Ottawa, Ontario, Canada

${ }^{5}$ Département D'Epidémiologie et de Biostatistiques, Ecole des Hautes Études en Santé Publique, Paris, France

${ }^{6}$ International Health Market Trends, Paris, France

${ }^{7}$ Edwards Lifesciences, Irvine, California, United States

${ }^{8}$ Cardiology, Bichat Hospital, Paris, France

${ }^{9}$ Cardiothoracic Surgery Department, Louis Pradel Hospital, Lyon-Bron, France

${ }^{10}$ University Paris VII, Faculté de Médecine Paris-Diderot, Paris, France

Contributors Conception and design, or analysis and interpretation of data: DM-Z, PC, J-FP, J-MT. Drafting the article or revising it critically for important intellectual content: DM-Z, PC, ME-S, IGB, VC, PV, TEF, BI, DG, J-FO, AV, TM. Final approval of the version to be published: DM-Z, PC. Contributors as being responsible for the overall content as guarantors: DM-Z.

Funding This study was funded through a research contract between International Health Market Trends and Edwards Lifesciences. DM-Z had full access to the data and performed the statistical analyses independently.

Competing interests $\mathrm{DM}-\mathrm{Z}$ is a consultant for Edwards Lifesciences, Mardil and Cardiawave and receives research grants from Edwards Lifesciences and Abbott vascular. PC is an Edwards Lifesciences employee. M-ES has received research grants from Edwards Lifesciences. PV is an Edwards Lifesciences employee. TEF is an Edwards Lifesciences employee. BI has received consultant fees from Edwards Lifesciences and speaker's fees from Boehringer Ingelheim and Novartis. J-F0 has received Consultant fees from Landanger, Delacroix-Chevalier and speaker's fees from Abbott, Medtronic, Sanofi. AV has received speaker's fees from Edwards Lifesciences.

Patient consent for publication Not required.

Ethics approval Ethical approval was not required as all data in the PMSI database are anonymised.

Provenance and peer review Not commissioned; externally peer reviewed. Data availability statement Data may be available on valid request.

Open access This is an open access article distributed in accordance with the Creative Commons Attribution Non Commercial (CC BY-NC 4.0) license, which permits others to distribute, remix, adapt, build upon this work non-commercially, and license their derivative works on different terms, provided the original work is properly cited, appropriate credit is given, any changes made indicated, and the use is non-commercial. See: http://creativecommons.org/licenses/by-nc/4.0/.

ORCID iD

David Messika-Zeitoun http://orcid.org/0000-0002-6278-5670

\section{REFERENCES}

1 Nkomo VT, Gardin JM, Skelton TN, et al. Burden of valvular heart diseases: a population-based study. Lancet 2006;368:1005-11. 
2 Dziadzko V, Dziadzko M, Medina-Inojosa JR, et al. Causes and mechanisms of isolated mitral regurgitation in the community: clinical context and outcome. Eur Heart J 2019;40:2194-202.

3 Dziadzko V, Clavel M-A, Dziadzko M, et al. Outcome and undertreatment of mitral regurgitation: a community cohort study. Lancet 2018;391:960-9.

4 Mirabel M, lung B, Baron G, et al. What are the characteristics of patients with severe, symptomatic, mitral regurgitation who are denied surgery? Eur Heart J 2007;28:1358-65.

5 Enriquez-Sarano M, Suri RM, Clavel M-A, et al. Is there an outcome penalty linked to guideline-based indications for valvular surgery? Early and long-term analysis of patients with organic mitral regurgitation. J Thorac Cardiovasc Surg 2015;150:50-8.

6 Gammie JS, Chikwe J, Badhwar V, et al. Isolated mitral valve surgery: the Society of thoracic surgeons adult cardiac surgery database analysis. Ann Thorac Surg 2018;106:716-27.

7 Gammie JS, Sheng S, Griffith BP, et al. Trends in mitral valve surgery in the United States: results from the Society of thoracic surgeons adult cardiac surgery database. Ann Thorac Surg 2009;87:1431-9. discussion 7-9.

8 Kilic A, Shah AS, Conte JV, et al. Operative outcomes in mitral valve surgery: combined effect of surgeon and hospital volume in a population-based analysis. J Thorac Cardiovasc Surg 2013;146:638-46.

9 Lazam S, Vanoverschelde J-L, Tribouilloy C, et al. TwentyYear outcome after mitral repair versus replacement for severe degenerative mitral regurgitation: analysis of a large, prospective, multicenter, International registry. Circulation 2017;135:410-22.

10 Vassileva CM, Mishkel G, McNeely C, et al. Long-term survival of patients undergoing mitral valve repair and replacement: a longitudinal analysis of Medicare fee-for-service beneficiaries. Circulation 2013;127:1870-6.

11 Braun J, van de Veire NR, Klautz RJM, et al. Restrictive mitral annuloplasty cures ischemic mitral regurgitation and heart failure. Ann Thorac Surg 2008;85:430-7. discussion 6-7.

12 Petrus AHJ, Dekkers OM, Tops LF, et al. Impact of recurrent mitral regurgitation after mitral valve repair for functional mitral regurgitation: long-term analysis of competing outcomes. Eur Heart J 2019;40:2206-14.

13 Acker MA, Parides MK, Perrault LP, et al. Mitral-valve repair versus replacement for severe ischemic mitral regurgitation. $N$ Engl J Med 2014;370:23-32

14 Ferket BS, Ailawadi G, Gelijns AC, et al. Cost-Effectiveness of mitral valve repair versus replacement for severe ischemic mitral regurgitation: a randomized clinical trial from the cardiothoracic surgical trials network. Circ Cardiovasc Qual Outcomes 2018;11:e004466.

15 Chikwe J, Toyoda N, Anyanwu AC, et al. Relation of Mitral Valve Surgery Volume to Repair Rate, Durability, and Survival. J Am Coll Cardiol 2017:69:2397-406.

16 Castillo JG, Anyanwu AC, Fuster V, et al. A near 100\% repair rate for mitral valve prolapse is achievable in a reference center: implications for future guidelines. J Thorac Cardiovasc Surg 2012;144:308-12.

17 Gillinov AM, Mihaljevic T, Javadikasgari $\mathrm{H}$, et al. Early results of robotically assisted mitral valve surgery: analysis of the first 1000 cases. J Thorac Cardiovasc Surg 2018;155:82-91.

18 Suri RM, Taggarse A, Burkhart HM, et al. Robotic mitral valve repair for simple and complex degenerative disease: midterm clinical and echocardiographic quality outcomes. Circulation 2015;132:1961-8.

19 Badhwar V, Rankin JS, He X, et al. The Society of thoracic surgeons mitral Repair/Replacement composite score: a report of the Society of thoracic surgeons quality measurement Task force. Ann Thorac Surg 2016;101:2265-71.

20 Bolling SF, Li S, O'Brien SM, et al. Predictors of mitral valve repair: clinical and surgeon factors. Ann Thorac Surg 2010;90:1904-12. discussion 12.

21 Gammie JS, O'Brien SM, Griffith BP, et al. Influence of hospital procedural volume on care process and mortality for patients undergoing elective surgery for mitral regurgitation. Circulation 2007;115:881-7.

22 Rankin JS, Grau-Sepulveda M, Shahian DM, et al. The Impact of Mitral Disease Etiology on Operative Mortality After Mitral Valve Operations. Ann Thorac Surg 2018;106:1406-13.
23 Rankin JS, Hammill BG, Ferguson TB, et al. Determinants of operative mortality in valvular heart surgery. J Thorac Cardiovasc Surg 2006;131:547-57.

24 Vassileva CM, McNeely C, Mishkel G, et al. Gender differences in long-term survival of Medicare beneficiaries undergoing mitral valve operations. Ann Thorac Surg 2013;96:1367-73.

25 Vassileva CM, McNeely C, Spertus J, et al. Hospital volume, mitral repair rates, and mortality in mitral valve surgery in the elderly: an analysis of US hospitals treating Medicare fee-for-service patients. $J$ Thorac Cardiovasc Surg 2015;149:762-8.

26 Treasure T, Bridgewater B, Gallivan S. Quality assessment of cardiac surgery in Britain. Dtsch Med Wochenschr 2009;134 Suppl 6:S237-9.

27 Anyanwu AC, Bridgewater B, Adams DH. The lottery of mitral valve repair surgery. Heart 2010;96:1964-7.

28 PMSI. Programme de médicalisation des systèmes d'informations (PMSI), 2012. Available: http://wwwdreessantegouvfr/ redressements-du-programme-de-medicalisation-des-systemes-dinformations-pmsi

29 CCAM. Classification commune des actes médicaux(CCAM), 2012 Available: http://wwwamelifr/accueil-de-la-ccam/trouver-un-acte/ indexphp

30 Charlson ME, Pompei P, Ales KL, et al. A new method of classifying prognostic comorbidity in longitudinal studies: development and validation. J Chronic Dis 1987;40:373-83.

31 Monteagudo Ruiz JM, Galderisi M, Buonauro A, et al. Overview of mitral regurgitation in Europe: results from the European registry of mitral regurgitation (EuMiClip). Eur Heart $J$ Cardiovasc Imaging 2018;19:503-7.

32 Goldstein D, Moskowitz AJ, Gelijns AC, et al. Two-Year outcomes of surgical treatment of severe ischemic mitral regurgitation. $N$ Engl J Med 2016;374:344-53.

33 Smith PK, Puskas JD, Ascheim DD, et al. Surgical treatment of moderate ischemic mitral regurgitation. $N$ Engl $\mathrm{J}$ Med 2014;371:2178-88.

34 Obadia J-F, Messika-Zeitoun D, Leurent G, et al. Percutaneous repair or medical treatment for secondary mitral regurgitation. $N$ Engl $J$ Med 2018;379:2297-306.

35 Stone GW, Lindenfeld J, Abraham WT, et al. Transcatheter mitral-valve repair in patients with heart failure. $N$ Engl J Med 2018;379:2307-18.

36 Detaint D, Sundt TM, Nkomo VT, et al. Surgical correction of mitral regurgitation in the elderly: outcomes and recent improvements. Circulation 2006;114:265-72.

37 Suri RM, Vanoverschelde J-L, Grigioni F, et al. Association between early surgical intervention vs watchful waiting and outcomes for mitral regurgitation due to flail mitral valve leaflets. JAMA 2013;310:609-16.

38 Enriquez-Sarano M, Akins CW, Vahanian A. Mitral regurgitation. Lancet 2009;373:1382-94

39 Mantovani F, Clavel M-A, Michelena HI, et al. Comprehensive Imaging in Women With Organic Mitral Regurgitation: Implications for Clinical Outcome. JACC Cardiovasc Imaging 2016;9:388-96.

40 Seeburger J, Eifert S, Pfannmüller B, et al. Gender differences in mitral valve surgery. Thorac Cardiovasc Surg 2013;61:42-6.

41 Baumgartner H, Falk V, Bax JJ, et al. 2017 ESC/EACTS guidelines for the management of valvular heart disease. Eur Heart $J$ 2017;38:2739-91.

42 Nishimura RA, Otto CM, Bonow RO, et al. 2017 AHA/ACC focused update of the 2014 AHA/ACC guideline for the management of patients with valvular heart disease: a report of the American College of Cardiology/American heart association Task force on clinical practice guidelines. Circulation 2017;135:e1159-95.

43 Vemulapalli S, Grau-Sepulveda M, Habib R, et al. Patient and hospital characteristics of mitral valve surgery in the United States. JAMA Cardiol 2019;4:1149-55.

44 lung B, Delgado V, Lazure P, et al. Educational needs and application of guidelines in the management of patients with mitra regurgitation. A European mixed-methods study. Eur Heart $J$ 2018;39:1295-303.

45 Gillinov M, Mick S, Suri RM. The specialty of mitral valve repair *. J Am Coll Cardiol 2017;69:2407-9. 